\title{
Direct adaptive inverse control
}

\author{
Muhammad Shafiq ${ }^{1 \mathrm{a})}$ and Muhammad A. Shafiq ${ }^{2 \mathrm{~b})}$ \\ ${ }^{1}$ International Islamic University, Islamabad, Pakistan \\ ${ }^{2}$ Ghulam Ishaq Khan Institute of Engineering Sciences and Technology \\ Topi, N.W.F.P, Pakistan \\ a)shafiqsaeeda@yahoo.com \\ b) amirshafiq@gmail.com
}

Abstract: In Adaptive Inverse Control (AIC), parameters of the inverse are obtained using Indirect method. In this paper, we propose a direct method to design adaptive inverse controller. Direct Adaptive Inverse Control (DAIC) alleviates the adhocism in adaptive loop. Direct and Indirect methods for adaptive inverse control are compared using computer simulations. DAIC shows better results compared to Indirect AIC in terms of tracking.

Keywords: Adaptive tracking, Direct Adaptive Inverse Control, Nonminimum phase systems

Classification: Electronic instrumentation and control

\section{References}

[1] B. Widrow and E. Walach, Adaptive Inverse Control: A Signal Processing Approach, Prentice Hall PTR, 1995, ISBN: 0130059684.

[2] B. Widrow and M. Bilello, "Adaptive Inverse Control," Proc. 1993 international symposium on Intelligent Control, Chicago, USA, pp. 1-6, 25-27, Aug. 1993.

[3] B. Widrow and G. L. Plett, "Adaptive Inverse Control based on Linear and Nonlinear Adaptive Filtering," IEEE Proc. 1996 International Workshop on Neural Networks for Identification, Control, Robotics, and Signal Image Processing (NICROSP '96), Venice, Italy, pp. 30-38, 21-23, Aug. 1996.

[4] L. Ming, Y. Cheng, and S. Yu, "An Improved Nonlinear Adaptive Inverse Control Systems Based on Filtered- $\epsilon$ LMS Algorithm," Proc. 27th Chinese control conference, Kunming, China, pp. 101-105, 16-18, July 2008.

[5] G. L. Plett, "Adaptive Inverse Control of Linear and Nonlinear Systems Using Dynamic Neural Networks," IEEE Trans. Neural Netw., vol. 14, no. 3, pp. 360-376, March 2003.

[6] F. M. Dias and A. M. Mota, "Direct Inverse Control of a Kiln," 4th Portuguese Conference on Automatic Control, Guimares, Portugal, 2000.

[7] A. M. Karshenas, M. W. Dunnigan, and B. W. Williams, "Adaptive inverse control algorithm for shock testing," IEE Proc. Control Theory and Applications, vol. 147, no. 3, pp. 267-276, May 2000.

[8] M. Shafiq, "Internal model control structure using adaptive inverse control strategy," ISA Trans., vol. 44, no. 3, pp. 353-362, June 2005. 


\section{Introduction}

AIC is a well established adaptive tracking methodology. Robust tracking and computationally less expensive characteristics of AIC has attracted the interest of many researchers for several decades $[1,2,3,4,5]$. AIC schemes are applicable to stable or stabilized plants [1]. AIC has been applied successfully to several practical applications such as Noise cancellation, Real-time blood pressure control, Control of kiln, Shock testing, Real-time control of temperature of a heating process and Real-time speed control of a brush DC motor etc $[1,6,7,8]$.

Discrete type Plants for which one or more zeros lie outside the unit circle are called Non-minimum phase plants. Numerous techniques have been developed for control of non-minimum phase plants. AIC based on linear and non-linear filtering, Non-linear Adaptive Inverse Control Systems Based on Filtered- $\epsilon$ LMS Algorithm, AIC of linear and non-linear systems using dynamic Neural-Networks and Internal model control structure using adaptive inverse control strategy are few of them $[2,3,4,5,8]$. Majority of control schemes for non-minimum phase plants are Indirect. In some AIC schemes inverse is designed based on identified plant. Most of AIC schemes estimate right inverse and then it is used as left inverse by considering left and right inverse are equal, but they are not equal, because practical plants most often have some kind of non-linearities. Right inverse means that inverse is estimated after the plant i.e. adaptive inverse filter follows plant. Since plant and its inverse are in cascade, they collectively form a unity gain transfer function. Similarly for the left inverse, inverse is estimated before plant i.e. adaptive inverse filter precedes plant. The algorithms in which right inverse is used as left inverse may not establish good tracking.

In this paper, we propose direct adaptive technique based on Normalized Least Mean Square (NLMS) for control of discrete time linear plants to alleviate the adhocism in adaptive loop. DAIC can be used for tracking of stable or stabilized, minimum or non-minimum phase discrete time linear plants. Little modification can also establish Model Reference Adaptive tracking.

\section{Problem statement}

Let us consider $P\left(q^{-1}\right)$ be a discrete time stable or stabilized linear plant. Let $P\left(q^{-1}\right)$ be given by

$$
\begin{gathered}
P\left(q^{-1}\right)=q^{-d} \frac{B\left(q^{-1}\right)}{A\left(q^{-1}\right)} \\
A\left(q^{-1}\right)=1+a_{1} q^{-1}+a_{2} q^{-2}+\ldots+a_{n} q^{-n} \\
B\left(q^{-1}\right)=b_{0}+b_{1} q^{-1}+b_{2} q^{-2}+\ldots+b_{m} q^{-m}
\end{gathered}
$$

Where, $q^{-1}$ is a back shift operator defined as $q^{-1} y(k)=y(k-1), k$ is positive integer that represents time instant, $d$ is a positive integer; it represents delay of the plant. $n$ and $m$ are positive integers and $n \geq m . A\left(q^{-1}\right)$ and $B\left(q^{-1}\right)$ are relatively co-prime polynomials. We also assume that plant may be nonminimum phase i.e. inverse of plant is unstable. Let $r(k), y_{d}(k)$ and $y(k)$ be 
the reference input, desired output and plant output respectively. Further, it is assumed that parameters of the plant are unknown or slowly time varying compared to the adaptation algorithm. The objective is to design a controller such that $y(k)$ tracks $y_{d}(k)$ i.e. $\lim _{k \rightarrow \infty}\left(e_{r e f}(k)\right)^{2}=\lim _{k \rightarrow \infty}\left(y_{d}(k)-y(k)\right)^{2}=$ $\epsilon$. Where, $y_{d}(k)=r(k-L), L$ is a positive integer that represents known delay. $e_{r e f}(k)$ is error at instant $k$ and $\epsilon$ is arbitrarily small positive real number in neighborhood of zero.

\section{Overview of indirect AIC scheme}

Control scheme for linear plants that uses Indirect AIC is introduced in [2]. Inverse control scheme for non-minimum phase plants introduced in [2] is shown in Fig. 1. Right inverse $\widehat{Q}_{R}\left(q^{-1}\right)$ is estimated using inverse model identification. The $\widehat{Q}_{R}\left(q^{-1}\right)$ is then copied into feed-forward path of plant i.e. $\widehat{Q}_{R \text { copy }}\left(q^{-1}\right)$. Delay is removed from Fig. 1 for controlling minimum phase plants [2]. $e_{r}(k)$ is used to adapt the weights of adaptive filter. When $e_{r}(k) \rightarrow$ 0 then $e(k)$ will also approaches to zero i.e.

$$
e(k)=\left[q^{-L}-\widehat{Q}_{R c o p y}\left(q^{-1}\right) P\left(q^{-1}\right)\right] r(k)
$$

Due to commutability of linear filters $\widehat{Q}_{R \text { copy }}\left(q^{-1}\right) P\left(q^{-1}\right) \cong P\left(q^{-1}\right) \widehat{Q}_{R}\left(q^{-1}\right)$. Indirect scheme discussed above estimates $\widehat{Q}_{R}\left(q^{-1}\right)$ and then it is copied in feed-forward path as left inverse $\widehat{Q}_{L}\left(q^{-1}\right)$. There are situation in which $\widehat{Q}_{\text {Rcopy }}\left(q^{-1}\right)$ may not be equal to $\widehat{Q}_{L}\left(q^{-1}\right)$ because of non-linearities in the plant. So, the use of $\widehat{Q}_{R \text { copy }}\left(q^{-1}\right)$ instead of $\widehat{Q}_{L}\left(q^{-1}\right)$ in such situations will not accomplish tracking.

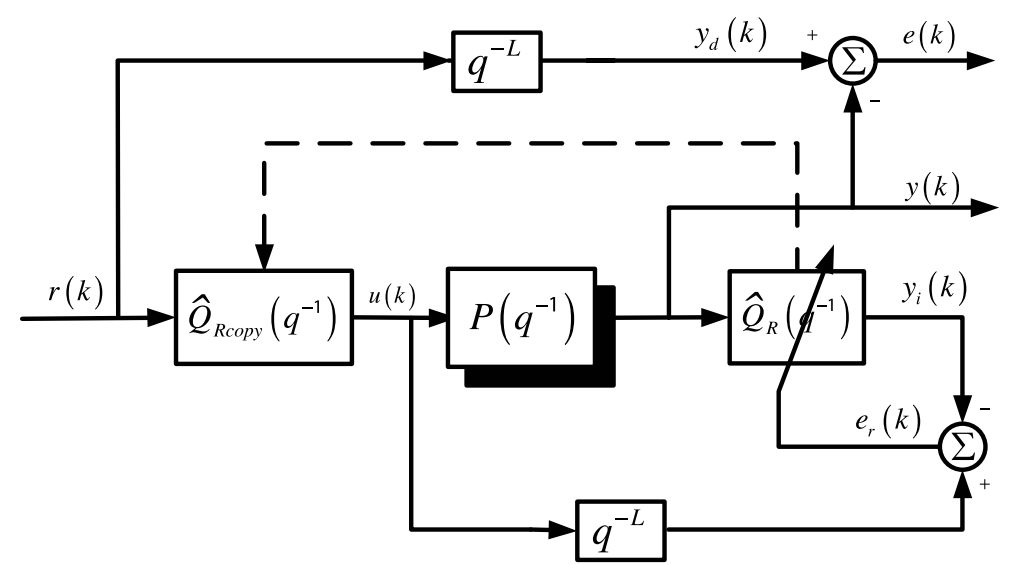

Fig. 1. Indirect control scheme for non-minimum phase plants.

\section{Design of DAIC}

We propose DAIC structure shown in Fig. 2. To the best of our survey, previous studies on AIC and DAIC have not discussed the scheme depicted in Fig. 2. In this structure, approximate inverse system $\widehat{Q}_{L}\left(q^{-1}\right)$ is directly 


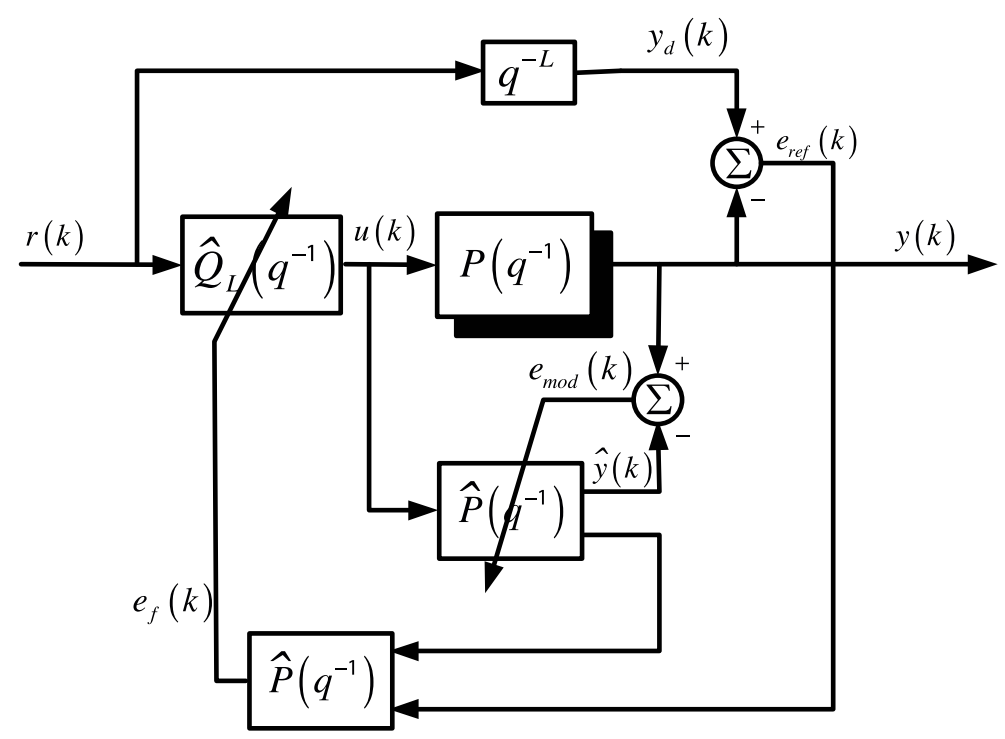

Fig. 2. Direct Adaptive Inverse Control scheme.

estimated. Control input to plant is synthesized by

$$
u(k)=\widehat{Q}_{L}\left(q^{-1}\right) r(k)
$$

The online estimation of $\widehat{Q}_{L}\left(q^{-1}\right)$ is accomplished using three steps given below:

1. Adaptive plant model $\widehat{P}\left(q^{-1}\right)$ is obtained using NLMS adaptive filter.

2. The mismatch error $e_{r e f}(k)$ between desired response $y_{d}(k)$ and plant output $y(k)$ is propagated through plant model $\widehat{P}\left(q^{-1}\right)$.

3. Output obtained from the second step $e_{f}(k)$, is used to adapt the weights of controller, which is also an NLMS adaptive filter.

In this algorithm the parameters of the controller $\widehat{Q}_{L}\left(q^{-1}\right)$ are estimated directly (i.e. $\widehat{Q}_{\text {Rcopy }}\left(q^{-1}\right)$ is not used), this is why we call it DAIC. Plant is preceded by the controller. There is no direct feedback from the plant output. Control scheme is not strictly feed-forward because controller weights are updated such that it contains information about the plant output and reference input. As shown in Fig. 2, we identify the plant as moving average system (i.e. The plant is approximated by an adaptive Finite Impulse Response (FIR) filter. As $k \rightarrow \infty$ then $e_{\text {mod }}(k) \rightarrow 0$, Where $e_{\text {mod }}(k)$ is error mismatch between plant output $y(k)$ and output of adaptive filter $\widehat{y}(k))$. Then for estimation of the adaptive inverse controller parameters $e_{f}(k)=\widehat{P}\left(q^{-1}\right) e_{r e f}(k)$ is used as error signal. We use NLMS algorithm to estimate the plant and adaptive inverse controller. Mean Square Error (MSE) between desired output and plant output for non-minimum phase plants can be made small by incorporating the delay $q^{-L}$. $\widehat{Q}_{L}\left(q^{-1}\right)$ is used as feed-forward controller for $P\left(q^{-1}\right)$. This gives $\widehat{Q}_{L}\left(q^{-1}\right) P\left(q^{-1}\right)=q^{-L} . q^{-L}$ is generally kept small for minimum phase and large for non-minimum phase plants.

Using $\widehat{Q}_{R}\left(q^{-1}\right)$ for $\widehat{Q}_{L}\left(q^{-1}\right)$ in Indirect AIC introduces atleast one step delay in the controller parameters. DAIC alleviates the adhocism of adaptive 
loop by directly incorporating an adaptive controller $\widehat{Q}_{L}\left(q^{-1}\right)$ in feed-forward loop. Since plant model is identified first, DAIC is less sensitive to plant uncertainties and variations. Further mild non-linearities at the output of plant may be learnt by $\widehat{Q}_{R}\left(q^{-1}\right)$ in Indirect AIC causing deviation from desired signal. Using $\widehat{Q}_{R \text { copy }}\left(q^{-1}\right)$ as left inverse may not then accomplish tracking as commutability is lost. DAIC is free from this deficiency. In DAIC $\lim _{k \rightarrow \infty}\left(e_{r e f}(k)\right)^{2} \rightarrow \epsilon$ provided $\lim _{k \rightarrow \infty}\left(e_{\text {mod }}(k)\right)^{2} \rightarrow \epsilon_{1}$. Where, $\epsilon_{1}$ is arbitrarily small positive real number in neighborhood of zero. Weight updation of plant model $\widehat{P}\left(q^{-1}\right)$ is done using Eq. 6

$$
\theta(k+1)= \begin{cases}\theta(k) & \text { if } \psi(k) \psi^{T}(k)=0, \\ \theta(k)+\mu_{1} e_{\text {mod }}(k) \frac{\psi(k)}{\psi(k) \psi^{T}(k)} & \text { if } \psi(k) \psi^{T}(k) \neq 0 .\end{cases}
$$

Where, $\theta(k)$ is parameter vector for $\widehat{P}\left(q^{-1}\right)$ defined as $\theta(k)=\left[\beta_{0}, \beta_{1}, \ldots, \beta_{M}\right]$. $\mu_{1}$ is learning rate and $0 \leq \mu_{1} \leq 1 . \psi(k)$ is regression vector defined as $\psi(k)=[u(k), u(k-1), \ldots u(k-M)] . \quad M+1$ are number of plant model parameters. Weight updation for controller is given by Eq. 7 .

$$
\omega(k+1)= \begin{cases}\omega(k) & \text { if } \varphi(k) \varphi^{T}(k)=0, \\ \omega(k)+\mu_{2} e_{f}(k) \frac{\varphi(k)}{\varphi(k) \varphi^{T}(k)} & \text { if } \varphi(k) \varphi^{T}(k) \neq 0 .\end{cases}
$$

Where, $\omega(k)$ is parameter vector for $\widehat{Q}_{R}\left(q^{-1}\right) . \mu_{2}$ is learning rate and $0 \leq$ $\mu_{2} \leq 1 . \psi(k)$ is regression vector defined as $\psi(k)=[r(k), r(k-1), \ldots r(k-$ $N)] . N+1$ are number of controller parameters.

\section{Simulation results}

Computer simulations of DAIC and Indirect AIC scheme are presented to show effectiveness of proposed scheme. Let us consider a disturbance free discrete time non-minimum phase linear plant having $A\left(q^{-1}\right)=1+0.05 q^{-2}+$ $0.05 q^{-3}+0.02 q^{-4}$ and $B\left(q^{-1}\right)=q^{-1}+3 q^{-2}+3.5 q^{-3}$. This is a stable non-minimum phase plant having zeros at $-1.50 \pm 1.1180 i$, poles at $0.25 \pm$ $0.3708 i$, and $-0.25 \pm 0.1936 i$. In this example, we choose $\mu_{1}=0.01$ and $\mu_{2}=$ 0.001. Similarly learning rate for Indirect AIC is chosen 0.001. Sampling time is chosen $0.001 \mathrm{sec}$.

Desired output $y_{d}(k)=r(k-12)$ tracking for the first 6 sec is shown in Fig. 3 (a) and for first $20 \mathrm{sec}$ (Amplitude 0 1) is shown in Fig. 3 (b). Plant output in DAIC has less overshoot and converges to desired output quickly compared to Indirect AIC. Tracking error depicted in Fig. 3 (c)-(d) has less amplitude and converges to zero faster in DAIC compared to Indirect AIC. MSE is less for DAIC compared to Indirect AIC and is shown in Fig. 3 (e)-(f). Control input shown in Fig. $3(\mathrm{~g})$ has less overshoot in DAIC and converges faster compared to Indirect AIC. Model identification error $e_{\text {mod }}(k)$ in DAIC converges to zero very quickly and is shown in Fig. $3(\mathrm{~h})$.

\section{Conclusion}

A direct controller based on NLMS for adaptive tracking of stable plants is proposed. Proposed scheme is applicable to both minimum and non- 


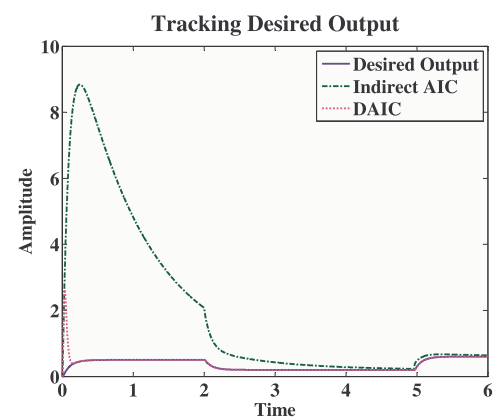

(a)

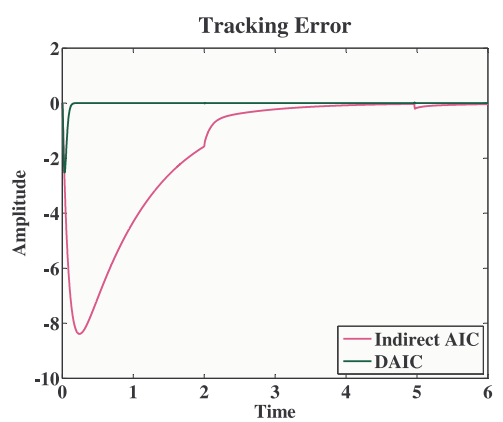

(c)

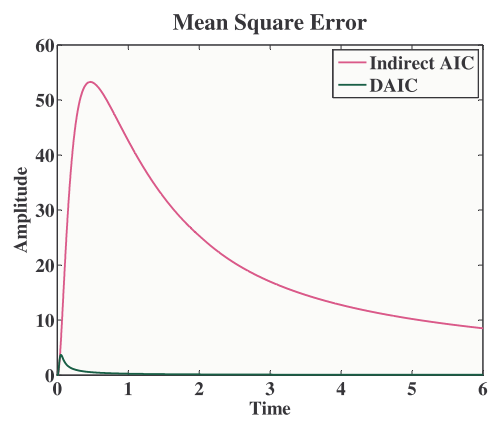

(e)

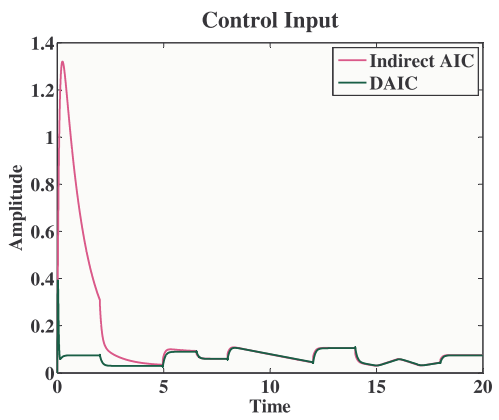

(g)

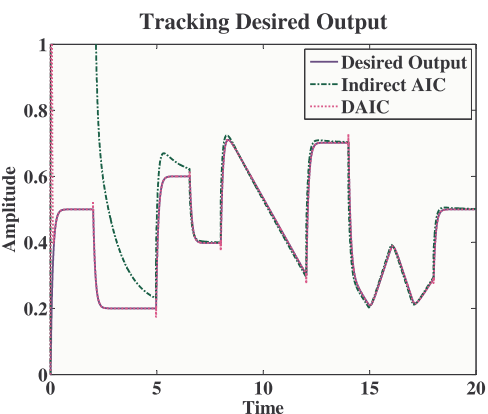

(b)

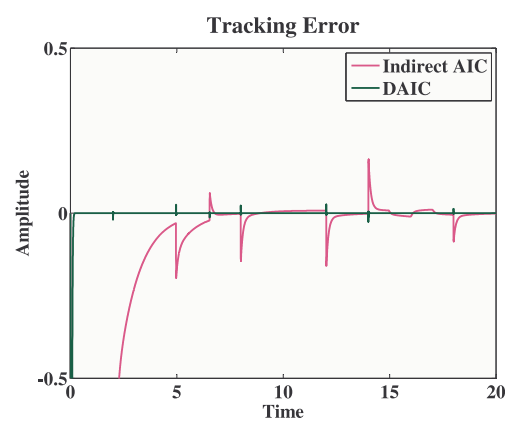

(d)

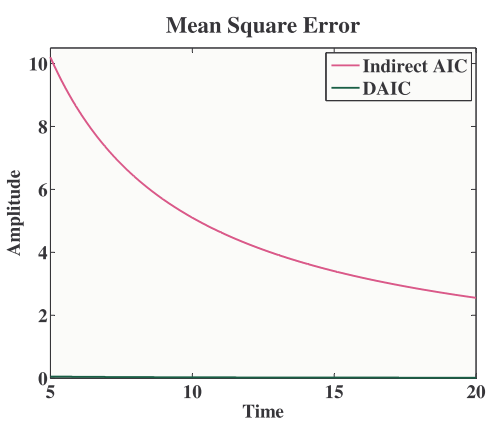

(f)

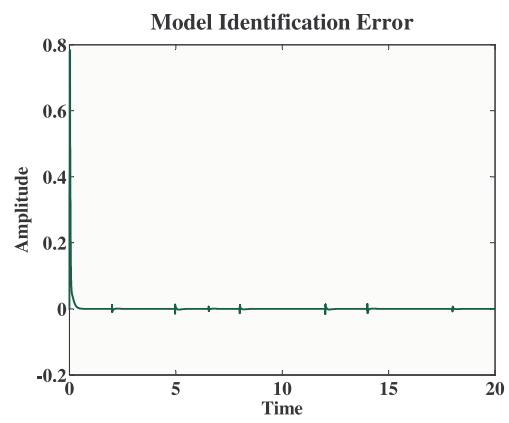

(h)

Fig. 3. Simulation results: (a) Tracking Desired Output (First $6 \mathrm{sec}$ ); (b) Tracking Desired Output (Amplitude 0 1); (c) Tracking error (First $6 \mathrm{sec}$ ); (d) Tracking error (Amplitude $-0.5 \sim 0.5$ ); (e) MSE (First $6 \mathrm{sec})$; (f) MSE (5 20 sec); (g) Control input; and, (h) Model Identification error. 
minimum phase discrete time linear plants. The inverse of the plant has been estimated directly in feed-forward loop as an adaptive FIR filter. NLMS algorithm is used for estimation of plant and controller. Simulation results shows that DAIC performs better than Indirect AIC in terms of Mean Square tracking error. 